\section{GENRE}

en séries

\section{Genre en séries}

Cinéma, télévision, médias

$6 \mid 2017$

Être mère, être père : représentations et discours médiatiques

\title{
Sébastien Hubier et Emmanuel Le Vagueresse (dir.), Gender et séries télévisées
}

Reims, Épure, 2016, 235 pages

\section{Barbara Dupont}

\section{OpenEdition \\ Journals}

Édition électronique

URL : http://journals.openedition.org/ges/1007

DOI : $10.4000 /$ ges. 1007

ISSN : 2437-6563

Éditeur

Presses universitaires de Bordeaux

\section{Référence électronique}

Barbara Dupont, « Sébastien Hubier et Emmanuel Le Vagueresse (dir.), Gender et séries télévisées »,

Genre en séries [En ligne], 6 | 2017, mis en ligne le 01 novembre 2017, consulté le 18 février 2021. URL : http://journals.openedition.org/ges/1007 ; DOl : https://doi.org/10.4000/ges.1007

Ce document a été généré automatiquement le 18 février 2021.

\section{cc) $(1) \odot$}

La revue Genre en séries est mise à disposition selon les termes de la Licence Creative Commons Attribution - Pas d'Utilisation Commerciale - Pas de Modification 4.0 International. 


\section{Sébastien Hubier et Emmanuel Le Vagueresse (dir.), Gender et séries télévisées}

Reims, Épure, 2016, 235 pages

\section{Barbara Dupont}

\section{RÉFÉRENCE}

Sébastien Hubier et Emmanuel Le Vagueresse (dir.), Gender et séries télévisées, Reims, Épure, 2016, 235 pages.

1 Avec Gender et séries télévisées, les codirecteurs Sébastien Hubier et Emmanuel Le Vagueresse proposent une incursion dans les représentations de genre se déployant dans les fictions sérielles, et témoignent ainsi de l'intérêt grandissant et nécessaire pour cette problématique dans le monde académique francophone. Le genre est ici entendu dans son acception systémique, devant donc être appréhendé dans son ensemble et son dynamisme. Ainsi, les dix auteur.e.s, joliment introduit.e.s comme des "yeux fertiles» (p. 14) percevant l'ouverture des possibilités narratives offertes par le récit sériel (et donc également l'altérité de genre qui peut s'y loger) jettent chacun.e un éclairage particulier sur le genre, approché tantôt dans sa construction, sa déstabilisation, sa crise parfois, ou encore les rapports entre ses pôles, féminin et masculin.

2 Bien que la table des matières ne propose pas de découpage thématique, la question du genre se décline de multiples façons au fil des articles qui laissent deviner en filigrane trois approches principales des représentations de genre : leur évolution, leur possible instabilité, et le statu quo que ces représentations permettent éventuellement de maintenir.

3 Les trois premiers textes se rejoignent en effet autour des (tentatives de) représentations changeantes du genre, particulièrement du féminin. Stella Louis se 
penche sur Buffy (1997-2003, The WB puis UPN), True Blood (2008-2014, HBO) et Once Upon a Time (2011-, $\mathrm{ABC}$ ) pour y décrypter les possibles évolutions du trope de l'héroïne blonde, jadis envoyée dans les ruelles sombres pour s'y faire tuer, et qui tente aujourd'hui de s'en détacher " pour nous offrir une redéfinition de la femme moderne " (p. 32). Sex and the City (1998-2004, HBO) et Desperate Housewives (2004-2012, ABC) sont ensuite approchées par Sébastien Hubier, qui s'intéresse à la hiérarchisation des rôles genrés à l'œuvre dans la fiction sérielle depuis ses débuts. Plus précisément, son étude insiste sur les ambiguïtés perceptibles dans ces séries dès qu'elles sont approchées, non pas pour ce qu'elles disent effectivement, mais pour la façon dont elles sont interprétées par leurs publics. Ainsi, l'auteur rappelle que Sex and the City et Desperate Housewives ont donné lieu, chacune de façon spécifique, à des lectures contradictoires des représentations genrées qu'elles proposent, notamment dans le milieu académique. Ainsi, Hubier les caractérise-t-il de récits post-féministes, un qualificatif ici prudemment dépolitisé, appréhendé dans une acception englobante, correspondant " conjointement à une institutionnalisation du féminisme et à une interrogation critique de la portée des discours féministes » (p. 43). Remedios Ponce González nous ramène ensuite en Europe avec la série historico-familiale espagnole Cuéntame cómo pasó (2001-, La1) dans laquelle le personnage de Mercedes Alcántara nous donne à voir son évolution en femme émancipée (ou qui, à tout le moins, tente de l'être) dans la transition démocratique post-franquiste. Si ces trois textes dévoilent des héroïnes confiantes et armées (parfois littéralement) pour s'épanouir dans la société contemporaine, les auteur.e.s en tirent invariablement des conclusions en demi-teintes, laissant apparaître une volonté émancipatrice pour les femmes, mais souvent désincarnée de toute revendication politique, une "'égalité sans se presser', c'est-àdire, sans vouloir changer la société dans son essence » (p. 71).

Un deuxième questionnement se dégage avec les quatre textes suivants, tous traversés par des images du genre déstabilisé, ébranlé, transgressé. Emmanuel Le Vagueresse s'attaque aux cultissimes Mystères de l'Ouest (1965-1969, CBS), pour dévoiler, sous ses airs résolument straight de western, une lecture homoérotique principalement investie par le «Surmâle » James West et son acolyte occasionnellement travesti, Artie Gordon. Charles Joseph fait ensuite dialoguer trois textes : la série Transparent (2014-, Amazon), l'argumentation de Derrida dans son texte la «Loi du Genre », et la poétique Folie du Jour de Maurice Blanchot (sur laquelle s'appuie déjà Derrida). Ainsi, tout comme Derrida, dans $L a$ Folie du Jour, Joseph s'attèle à faire «intervenir la problématique sexuée au travers du grammatical dont fait usage le texte » (p.138) de Transparent. Le personnage principal de la série, Morton qui devient Maura, et sa façon de se raconter, sont alors mobilisés pour révéler que la loi du genre « est une loi en cours d'écriture dont chacun est son propre auteur » (p. 139). Dès lors, les genres, à la fois identitaires et littéraires, peuvent se jouer des perceptions pour mettre en lumière « des personnages qui sont, avant d'être homme ou femme, des individus, des personnes qui de fait construisent leur je-nre comme bon leur semble» (p.148). Ensuite, dans un texte se distinguant par son niveau d'abstraction du reste de l'ouvrage, Angel Delrez propose un mariage entre conceptualisation philosophique du féminin et écriture sérielle: il convoque en effet Lyotard, Benjamin, Bourdieu, mais surtout (à nouveau) Derrida pour élaborer une étude minutieuse de la séquence d'ouverture de la série chirurgicale de Steven Soderbergh, The Knick (2014-2015, Cinemax). Tant par le fond que par la forme que prend cet essai, l'auteur s'adosse à la lecture d'Eperons, les styles de Nietzsche, dans lequel Derrida lie la question du style à celle du féminin. En dépliant minutieusement 
cette scène, dans laquelle un médecin sectionne le ventre d'une femme enceinte présentant une anomalie placentaire, Angel Delrez s'applique à son tour à dévoiler la congruence entre le style (de l'écriture écranique, cette fois) et le féminin. Ainsi, l'auteur s'intéresse-t-il d'une part à ces premiers coups de bistouri sur le corps de la femme enceinte, qui semblent fonctionner comme une «trans-scription sérielle 'du découpage lui-même'« (p.153), révélant l'incisivité propre à l'opération filmique. D'autre part, il aborde ce « corps-enceinte » (p. 155) qu'il nous enjoint à lire comme une métonymisation du film, « un ensemble rassemblé et fécond qui va transmettre ce qu'il véhicule au lieu de le perdre » (Lyotard, cité par Delrez, p. 154). Antonio Domínguez Leiva se penche ensuite sur l'éroticisation de l'exotisme, de l'altérité permise par le genre science-fictionnel dans la série spatiale Star Trek: The Original Series (1966-1969, $\mathrm{NBC}$ ) tant à travers ses personnages féminins (notamment à travers la sexy little green girl) que masculins (dont Captain Kirk représentera le paroxysme de l'hétéronormativité). Si la porte s'ouvre, à travers ces quatre textes, sur le genre pensé hors des carcans normatifs solidement fixés par la logique patriarcale, il est intéressant de noter que le genre fictionnel de la série, le genre en tant que catégorie typologique, accompagne aussi la réflexion des auteur.e.s et interroge dès lors immanquablement le rôle des spectateur.trices dans la perception et donc la construction du genre à l'écran.

Enfin, le troisième ensemble se dessine avec le dernier trio d'articles autour des normes et des stéréotypes de genre. Julio de los Reyes Lozano s'y intéresse d'abord aux TAV, les processus de traduction audiovisuelle auxquels les séries de langue étrangère sont systématiquement soumises en arrivant chez nous. Se concentrant sur un épisode de Mad Men (2007-2015, AMC), l'auteur compare le doublage français et espagnol d'une part, et la version originale d'autre part, afin d'y déceler « les différentes stratégies et techniques de traduction des éléments à caractère sexiste» (p. 203). Forte d'une méthodologie méticuleuse, l'analyse s'articule autour de trois types d'éléments: les mots et expressions à caractère sexuel, les stéréotypes de genre, et les compliments et insultes envers les individus de l'autre sexe. À l'issue de sa démarche, dans les trois versions de l'épisode étudié, l'auteur constate le maintien, voire le renforcement de la connotation sexiste des échanges. Victor-Arthur Piégay prend ensuite le relais avec la série Charmed (1998-2006, The WB). En relevant les clichés et stéréotypes de genre à l'œuvre dans la série, il envisage notamment celle-ci comme présentant une «version consumériste et capitaliste de la troisième vague, quasi intégralement vidée de son contenu politique et contestataire » (p. 208). Enfin, Jennifer Houdiard clôture l'ouvrage par une étude de la figure paternelle présente dans les sitcoms familiales catalanes Majoria absoluta (2002-2004, TV3) et La sagrada família (2010-2011, TV3). Si les deux séries divergent sur plusieurs points, elles se rejoignent à des degrés divers dans les modèles de virilité qu'elles donnent à voir. En effet, de la masculinité hégémonique à la figure du macho ibérico, l'auteure décèle dans ces représentations catalanes un héritage étasunien et espagnol et confirme que le ton de l'humour ne suffit pas à déconstruire les clichés dans ces séries. En soulignant le statu quo, à différents niveaux, des représentations sexistes et des stéréotypes de genre dans les séries TV, ces trois analyses pointent également plusieurs zones potentielles de renforcement, ou à l'inverse d'ébranlement, de ces derniers: ainsi, les stratégies de traduction, les influences étrangères ou encore le rôle essentiel du public dans sa compréhension du texte sériel se révèlent comme des enjeux centraux de la construction du genre dans les séries TV contemporaines et futures. 
6 La diversité des objets et des approches prête à cet ouvrage un caractère surprenant et éclaté, invitant le lecteur d'un cadre à l'autre dans une flexibilité stimulante. La souplesse des lectures est nécessairement concomitante d'une transdisciplinarité assumée par les directeurs de l'ouvrage dès les premières pages, mais elle s'accompagne aussi inévitablement d'une versatilité méthodologique ne laissant finalement que peu d'espace aux outils et méthodes spécifiques de l'étude des séries télévisées. En effet, ces dernières semblent fonctionner davantage comme un prétexte que comme un point focal (la plupart des articles font d'ailleurs davantage référence aux études filmiques pour ancrer leur propos), comme semble l'annoncer la bibliographie sélective proposée par les codirecteurs dès la préface. L'ouvrage se découvre alors comme une collection de fragments captivants sur les représentations du genre, reléguant au second plan l'héritage littéraire des television studies et TV series studies. Par ailleurs, l'approche gender, deuxième liant du livre, souffre par moments d'une certaine forme de prudence, qui tend à maintenir le genre dans le périmètre du binaire et de l'attendu. Toutefois, bien que ces précautions affaiblissent possiblement le caractère novateur de l'ouvrage, elles attestent aussi de la volonté des codirecteurs d'organiser une diversité notable de corpus en un ensemble unifié et cohérent. Ainsi, si certains manquements peuvent être soulignés, tant en ce qui concerne les fictions sérielles que l'approche du genre, Gender et séries télévisées témoigne avant tout de la complexité et de la richesse des deux domaines que l'ouvrage se propose de conjuguer. Il en offre en effet un aperçu forcément partiel, mais aussi ambitieux et vivement pertinent dans la recherche francophone. 